\title{
Electric Charge Quantization and Extra Dimensions
}

\author{
A. Pérez-Lorenzana ${ }^{1,2 *}$ and C. A. de S. Pires ${ }^{3 \dagger}$ \\ 1 The Abdus Salam International Centre for Theoretical Physics, I-34100, Trieste, Italy \\ ${ }^{2}$ Departamento de Física, Centro de Investigación y de Estudios Avanzados del I.P.N. \\ Apdo. Post. 14-740, 07000, México, D.F., México \\ ${ }^{3}$ Departamento de Física, Universidade Federal da Paraíba, \\ Caixa Postal 5008, 58051-970 João Pessoa PB, Brazil.
}

(May, 2002)

In models with flat extra dimensions tiny Dirac neutrino masses can be generated via the coupling of four dimensional Standard Model fields to a higher dimensional fermion. Here we argue that, in spite of the Dirac nature of the neutrino, quantization of the electric charge can still be understood as a result of anomaly cancellation, charge conservation and naturalness requirements.

14.60.Pq; 14.60.St;11.10.Kk

\section{INTRODUCTION}

One of the most intriguing mysteries of nature is the empirical observation that the electric charge of the fundamental particles appears to be quantized. This question has already attracted some attention in the past and some possible ways to understand it are already known. The first suggestion was given by Klein [1] at the beginning of the past century through the introduction of extra dimensions. Another proposal came from Dirac [2] who linked electric and magnetic monopole charges. A third possibility arises in the context of Grand Unification Theories [3] where the quantization of charge comes naturally as a plus from the group structure.

More recently, in the context of four dimensional theories, some further attempts to understand the basics of this problem have addressed the question in a more general framework [4-6]: In a large class of models that include a U(1) gauge group factor contributing to electric charge, its quantization is not always guaranteed. The problem is that the $\mathrm{U}(1)$ generator can a priori take a continuous set of values. However, classical as well as quantum constraints may restrict such values. Namely, the requirement of having massive fermions and anomaly cancellation may, for instance, explain the values of the hypercharge taken by matter fields in a single family in the Standard Model (SM). Though one may take this as a cheerful notice, two facts should be yet considered. First, in the SM neutrinos are massless, which alone seems not to be in good agreement with the observational evidence of neutrino oscillations in solar [7], atmospheric [8] and terrestrial [9] neutrino experiments. Second, unless one makes the extra assumption that all families are exact replicas of each

\footnotetext{
*e-mail:aplorenz@ictp.trieste.it

†e-mail:cpires@ift.unesp.br
} 
other, in the sense that similar representations have equal hypercharge, the electric charge quantization in the actual SM with three families seems again ad hoc. In fact, in this case there exist some hidden symmetries which, being anomaly free, insert an arbitrariness in the definition of the electric charge $[4,5]$. In order to restore electric charge quantization one has to break those hidden symmetries. The simplest way to do it is by given the neutrino a Majorana mass [5]. Indeed, if neutrinos are of a Dirac type the electric charge appears to be dequantized. This is due to the introduction of extra degrees of freedom, the right handed neutrinos, which come with out adding extra constraints to the theory.

In this short note we are interested in analyzing these results in the context of theories that involve large extra dimensions [10]. We are mainly motivated by the fact that Dirac neutrinos are a very likely byproduct of such theories [11-14]. Our main observation here is as follows: Even though at first sight a Dirac neutrino does not seem to go well with electric charge quantization, in models with extra dimensions the requirement of charge conservation and naturalness on the explanation to neutrino masses introduce an extra constraint on the assignment of hypercharges that leads to the restoration of electric charge quantization. Shortly, they fix the hypercharge of the right handed neutrinos to be null. This is a dynamical version of the same condition introduced when one writes (by hand) Majorana mass terms for the neutrinos. To make our point, and for completeness, we shall first review the electric charge quantization in the usual four dimensional SM with one family, and explicitly show how the introduction of a right handed neutrino state with only a Dirac coupling dequantize the electric charge. Next, we shall analyze the case of models with extra dimensions and argue that here, even if neutrinos are Dirac fields, the electric charge quantization is restored, provided that the right handed neutrino is a bulk field. Further remarks on these theories are added by the end.

\section{CHARGE QUANTIZATION AND NEUTRINO MASS}

Let us start by reviewing the well known case of the SM with a single family. Due to the abelian nature of hypercharge, $Y$, the assignment of charges to matter fields is in principle arbitrary. However, symmetry breaking triggered by the acquisition of a vev by the Higgs doublet, $H$, relates $Y$ with electric charge through the formula

$$
Q=T_{3 L}+\frac{1}{2} \frac{Y}{Y_{H}}
$$

where $Y_{H}$ is the $H$ hypercharge, which can always be normalized to $Y_{H}=1$. For the fermion sector we take

$$
\begin{array}{llll}
Q_{L}: & \left(3,2, Y_{q}\right), & u_{R}: & \left(3,1, Y_{u}\right), \\
d_{R}: & \left(3,1, Y_{d}\right), & L: & \left(1,2, Y_{\ell}\right), \\
e_{R}: & \left(1,1, Y_{e}\right) . & &
\end{array}
$$

Now, the requirement of renormalizability of the gauge theory, as well as the existence of Higgs couplings that generate fermion masses, introduce a number of constraints among the above hypercharges that further reduce their arbitrariness. We first consider the Yukawa coupling terms 


$$
\mathcal{L}_{Y}=h^{\ell} \bar{L} H e_{R}+h^{u} \bar{Q}_{L} \tilde{H} u_{R}+h^{d} \bar{Q}_{L} H d_{R}+\text { h.c. }
$$

They give

$$
Y_{e}=Y_{\ell}-1 ; \quad Y_{u}=1+Y_{q} ; \quad \text { and } \quad Y_{u}=Y_{q}-1 .
$$

Cancellation of the triangular quantum anomalies, namely $\operatorname{Tr} U(1)_{Y}\left[S U(3)_{c}\right]^{2}$; $\operatorname{Tr} U(1)_{Y}\left[S U(2)_{L}\right]^{2} ; \operatorname{Tr}\left[U(1)_{Y}\right]^{3} ;$ and $\operatorname{Tr} U(1) \times$ grav. anomaly; contribute with only two independent constraints, which combined with those above can be written as

$$
\begin{aligned}
\operatorname{Tr} U(1)_{Y}\left[S U(2)_{L}\right]^{2} & \Rightarrow 3 Y_{q}+Y_{\ell}=0 ; \\
\text { and } \operatorname{Tr}\left[U(1)_{Y}\right]^{3} & \Rightarrow Y_{\ell}=-1 .
\end{aligned}
$$

Other anomalies are identically canceled with the help of the above conditions. Notice that there are as many constraints as free parameters in our analysis. Therefore, a unique solution exist which gives the well known values:

$$
\begin{array}{ll}
Y_{q}=1 / 3 ; & Y_{u}=4 / 3 ; \quad Y_{d}=-2 / 3 ; \\
Y_{\ell}=-1 ; & Y_{e}=-2 .
\end{array}
$$

Hence, this procedure provides a natural explanation to quantization of charge. Nevertheless, as already pointed out, this theory contains only massless neutrinos.

\section{A. Dequantization by a Dirac neutrino}

By minimally extending the spectrum of the theory to contain a right handed weak and color fermion singlet, $\nu_{R}:\left(1,1, Y_{\nu}\right)$, we allow for a new Yukawa coupling of the form $L H \nu_{R}$. Thus, besides the constraints in Eq. (3) we now also have

$$
Y_{\nu}=1+Y_{\ell} .
$$

However, now anomaly cancellation yields to only one extra constraint on $Y_{\ell}$ and $Y_{q}$, the one associated to $\operatorname{Tr} U(1)_{Y}\left[S U(2)_{L}\right]^{2}: 3 Y_{q}+Y_{\ell}=0$. All other anomaly expressions become just identities. Therefore, such theory still has one free parameter, $Y_{\ell}$, which can be fixed arbitrarily. This spoils the quantization of the electric charge. This phenomenon is usually referred as charge dequantization and it is associated to the presence of some hidden (anomaly free) global symmetry in the theory $[4,6]$, here identified as $U(1)_{B-L}$.

If the theory is assumed to be an effective theory in which a Majorana mass term, $\bar{\nu}_{R}^{c} \nu_{R}$, is present, this adds the lacking constraint, fixing the extra degree of freedom, by explicitly breaking the $U(1)_{B-L}$ symmetry. Clearly, writing this term is equivalent to take $Y_{\nu}=0$. This fixes $Y_{\ell}=-1$, and other charges follow as needed.

\section{B. Dequantization with three families}

The above analysis can be straightforwardly generalized for three families. Notice, however, that by writing the Yukawa couplings one should keep in mind that there is a mixing in the quark sector, parametrized by the CKM matrix. Thus, one gets 


$$
\mathcal{L}_{Y}=h_{i i}^{\ell} \bar{L}_{i} H e_{i R}+h_{i j}^{u} \bar{Q}_{i L} \tilde{H} u_{j R}+h_{i j}^{d} \bar{Q}_{i L} H d_{j R}+\text { h.c. }
$$

From here, the hypercharges of the quark fields should obey $Y_{u i}=Y_{u} ; Y_{d i}=Y_{d}$ and $Y_{q i}=Y_{q}$. This reduces the number of constraints to $Y_{u}=Y_{q}+1 ; Y_{d}=Y_{q}-1$ and $Y_{e i}=Y_{\ell i}-1$; for the family indices $i, j=1,2,3$. Once more, requiring cancellation of anomalies is not enough as to uniquely define the hypercharges. As before, only $\operatorname{Tr} U(1)_{Y}\left[S U(2)_{L}\right]^{2}$; and $\operatorname{Tr}\left[U(1)_{Y}\right]^{3}$ give non trivial conditions, which added to those above may only fix seven of the remaining nine free parameters. Again, the charge is not quantized. The hidden symmetries are those associated to the lepton number combinations: $L_{e}-L_{\mu} ; L_{e}-L_{\tau} ; L_{\mu}-L_{\tau}$. The simplest way to break these symmetries is allowing for a general mixing in the leptonic sector as we did for the quark sector, since their presence adds extra constraints, namely $Y_{e i}=Y_{e}$, and $Y_{\ell j}=Y_{\ell}$. Thus, reducing the problem to the single family case. Such mixings appear naturally if neutrinos are massive, but again, if neutrinos are Dirac-like, $B-L$ will reappear as a hidden symmetry that, once more, plays against charge quantization.

\section{BULK NEUTRINO AND CHARGE QUANTIZATION}

\section{A. Neutrino mass in extra dimensions}

All above observations seem not to go well with the possibility that the neutrinos be Dirac-like particles. At first glance this is a good motivation to assume that they are rather Majorana particles. A second motivation for a Majorana neutrino comes from the fact that a Dirac mass needs a large fine tuning to provide $\mathrm{eV}$ masses, whereas Majorana masses can be explained quite naturally by the see-saw mechanism [15]. So, most of the current models for explaining neutrino masses and mixings follow this trend.

Nevertheless, in theories with extra compact dimensions the situation is on the opposite. In those models SM particles are assume to live on a four dimensional hypersurface (the brane) embedded in a higher dimensional space (the bulk). The extra dimensions are here taken to be compactified on a flat manifold [10]. These theories have been motivated by the possibility of having a small fundamental scale for quantum gravity. The aftermath of such constructions is the reduction of the energy scale cut-off that suppresses all non renormalizable operators that involve SM particles, as for instance the dimension 5 operator that gives a Majorana mass to the neutrino:

$$
\frac{(L H)^{2}}{\Lambda} .
$$

The physical meaning of the scale $\Lambda$ depends on the nature of the compactification as well as on the physics that generates such an operator. In theories with flat extra dimensions, $\Lambda \leq M$, where $M$ is the fundamental scale at which gravity becomes strong, that related to the effective Planck scale $M_{P}$ and the volume of the n-th dimensional extra compact space, $V_{n}$, by the relationship [10]: $M^{n+2} V_{n}=M_{P}^{2}$. Current limits indicate that $M$ could be as low as few $\mathrm{TeV}[16,17]$.

With such a small $\Lambda$ the neutrino mass generated by the operator in Eq. (7) comes out to be too large. Thus, such operators have to be avoided, say, by imposing lepton number conservation. Instead, naturally small Dirac masses may be generated by introducing a bulk 
neutrino [11]. Now, despite of having only Dirac neutrinos, our previous understanding of the electric charge quantization can remain due to the own characteristics of these models.

To illustrate our claim let us briefly mention how light Dirac neutrinos are introduced in these theories. As a consequence of the localization of the SM fields on the brane, all their couplings to fields that freely propagate in the whole space get a volume suppression, so that they become very small in the effective four dimensional theory. This is the reasoning applied for understanding the smallness of neutrino mass. Let us consider a right handed bulk neutrino, $\nu_{B R}$, that couples to the SM fields through the Yukawa coupling

$$
\int d^{4} x d^{n} y \frac{\tilde{h}}{\sqrt{M^{n}}} \bar{L}(x) \tilde{H}(x) \nu_{B R}(x, y) \delta^{n}(y),
$$

with $\tilde{h}$ the Yukawa coupling. There we have assumed that the SM brane is localized at the position $y=0$, where $y$ represents the $n$ extra space coordinates. This coupling explicitly conserves total Lepton number and gives the same constraint for the hypercharges as in Eq. (6). The zero mode wave function of the bulk neutrino field goes as $\nu_{0 B R}(x) / V_{n}^{1 / 2}$. Then, after integrating out the extra dimensions and setting in the Higgs vev, $v$, one gets a small Dirac mass given as

$$
m_{D}=\tilde{h} \frac{M v}{M_{P}}
$$

Notice that for $M \sim 1 \mathrm{TeV}$ one gets a mass of order $10^{-5} \mathrm{eV}$ [10] for $\tilde{h}=1$. A larger fundamental scale, however, or moderately strong bulk coupling would produce the right order of masses for explaining neutrino anomalies. Moreover, requiring that $m_{D} \lesssim .1 \mathrm{eV}$ to account for the range of mass parameters needed to fit the experiment, one arises to the condition that

$$
\tilde{h}=\frac{M_{P} m_{D}}{M v} \lesssim 10^{-12}\left(\frac{M_{P}}{M}\right)
$$

\section{B. Naturalness and charge quantization}

There is a crucial ingredient in the scenario we are considering: SM gauge interactions are attached to the brane. In such an case any bulk field should be neutral under the SM groups, including the hypercharge. This is to insure the gauge invariance of the theory. In other words, if we allow a hypercharged field to propagate in the bulk, by assuming that our right handed neutrino has a non zero $Y_{\nu}$ for instance, then, we would be forced to promote the hypercharge to be a bulk symmetry. Here, a comment is in order. With $U(1)_{y}$ in the bulk, the radius of the extra space can not be larger than few hundred $\mathrm{GeV}^{-1}$, which makes the fundamental scale much larger, say than $10^{7} \mathrm{GeV}$ for $n=6$ to $10^{1} 3 \mathrm{GeV}$ for $n=1$. With such a large scale one may doubt about the need of the bulk neutrino, since the suppression on the operator in Eq. (7) is now much less sever. It is interesting, however, to analyze whether even under this circumstances the bulk neutrino hypothesis can still be at work to provide light Dirac neutrinos. This program immediately fails due to the volume suppression in bulk-brane couplings, which implies that the bulk theory should be strongly coupled. To 
clarify this point let us consider the coupling of the $U(1)_{Y}$ gauge field, $B_{\mu}(x, y)$, to the lepton current $j_{\mu}(x)=\bar{L} \gamma_{\mu} L$, which has the form

$$
\frac{g_{5}}{\sqrt{M^{n}}} Y_{\ell} B_{\mu}(x, y) j^{\mu}(x) \delta^{n}(y)
$$

In the above formula the suppression comes due to the larger mass dimensionality of the Gauge field: $[B]=1+n / 2$, which is reflected on the scaling of the Kaluza Klein modes. At the zero mode level one gets $B_{\mu}(x, y)=B_{\mu}^{0}(x) / \sqrt{V_{n}}+$ (higher levels). Therefore, after dimensional reduction one obtains an effective four dimensional theory where the coupling constant of the hypercharge is given by

$$
g=\left(\frac{M}{M_{P}}\right) g_{5}
$$

As one knows that $g$ is close to one, it comes that $g_{5}$ has to be large enough as to absorb the suppression on the right hand side.

Solving the neutrino mass problem with bulk neutrinos, however, needs an unnaturally large hierarchy between $g_{5}$ and $\tilde{h}$. In fact, by looking at Eqs. (9) and (11) one gets

$$
\frac{\tilde{h}}{g_{5}} \sim \frac{m_{D}}{g v} \lesssim 10^{-12} ;
$$

which is the same hierarchy one wished to explain since the beginning, independently of the actual value of the fundamental scale. In other words, promoting the hypercharge to be a bulk interaction jeopardizes our former explanation of the neutrino masses with a bulk neutrino. Hence, the consistency of the theory requires that the hypercharge should be confined to the brane as well as the other SM gauge interactions. Moreover, as the right handed neutrino would still come from the bulk, it would be forced to be totally chargeless under any SM group in order to keep gauge invariance.

As it is clear, above arguments mean that $Y_{\nu}=0$. This adds the missing constraint to the system of Eqs. (3) and (6), obtained from Yukawa couplings and anomaly cancellation conditions. Straightforwardly one gets, from Eq. (6), that $Y_{\ell}=-1$, which gives the right values of $Y$ as in Eq. (5). Therefore, our understanding of quantization of charge would remain though the neutrino is Dirac particle. Considering three families will give us the same answer.

\section{OUTLOOK}

\section{A. neutrino puzzles}

In connection with the possible explanation of neutrino anomalies, we would like to add few comments here. First, our whole discussion is only addressing the problem of preserving electric charge quantization given the presence of only Dirac mass terms on these theories. A simple and consistent explanation of both, solar and atmospheric neutrino data is actually possible in the present context $[12,13]$, though the theory will require the existence of at least two bulk neutrinos to accommodate the two required mass parameters. However, an 
explanation to LSND results is excluded [12], at least in the minimal version of these models (with three right handed neutrinos), since only two independent squared mass differences can be produced out of three mass eigenstates. Nevertheless, there is still the possibility that a fourth bulk neutrino exist that could provide the extra degree of freedom to accommodate LSND, as already suggested in Ref. [14] Notice that this conclusion is regardless the actual size of the common radius $R$ in theories with large extra dimensions $[12,13]$, mainly because Dirac mass terms only depend on the ratio $M / M_{P}$. Another consequence of these scenario would be the non observation of neutrinoless double beta decay.

\section{B. An anomalous B-L?}

As a final note, we observe that $B-L$ does not trivially arise as a gaugeable (anomaly free) symmetry any more. The clear example is the (simplest) case with one large extra dimension compactified on $S^{1}$. There, the bulk theory is vector like. For higher dimensions one has to cancel all anomalies in the bulk to keep gauge invariance. This usually needs the introduction of new extra fields. In the simplest case we would just assume that the bulk theory is vector-like, independently of the number of extra dimensions. Thus, there would no contribution to $B-L$ anomaly coming from bulk fields. However, with only the SM particle content, $B-L$ is anomalous. Indeed, for SM matter fields one has $\operatorname{Tr} U(1)_{B-L}^{3}=-3$. Such anomaly appears localized on the fixed point where the SM lives.

The naive approach of introducing orbifolds to project out chiral zero mode components of the bulk neutrino does not seem to help in canceling the $B-L$ anomaly. Indeed, in five dimensions, for instance, such theory develops localized anomalies that sit on the fixed points of the orbifold. Such anomalies, however, do not compensate the one developed by the SM fields [18], making the whole theory non gauge invariant under $B-L$. In fact, with a $Z_{2}$ orbifolding of the circle under which $y \rightarrow-y$, the bulk neutrino transforms as $\nu_{B} \rightarrow \gamma_{5} \nu_{B}$. Thus, at the fixed point $y=0, \nu_{B L}$ vanishes. Nevertheless, it also thus at the other fixed point at the end of the space, located at $y=\pi$. This accidental symmetry of the bulk $\nu_{B L}$ field is reflected in the 5D anomaly, which is actually localized at the orbifold fixed points:

$$
\partial_{M} J^{M}(x, y)=\frac{1}{2}[\delta(y)+\delta(y-\pi)] \mathcal{A}(x, y)
$$

where $J^{M}$ is the 5D current and $\mathcal{A} \sim F \cdot \tilde{F}$ is the $4 \mathrm{D}$ chiral anomaly. The SM anomaly contribution, on the other hand, would be

$$
\partial_{M} J^{M}(x, y)=-\delta(y) \mathcal{A}(x, y)
$$

which obviously does not compensate the 5D contribution. There is, of course the possibility of removing such an anomaly by increasing the number of bulk fields. However this may also affect our above understanding of charge quantization by the introduction of new degrees of freedom. A further analysis of this problem may deserve a further study. 


\section{CONCLUDING REMARKS}

Along this short note we have argued that, in the context of models with large compact extra dimensions, Dirac-like neutrinos are consistent with our understanding of electric charge quantization from gauge anomaly cancellation arguments. The reason is twofold: First, naturalness argument on the smallness of the neutrino mass, as generated via bulkbrane couplings, force all SM gauge interactions to be fixed to the brane. Second, in order to get a consistent gauge invariant theory, bulk fermions must be neutral under all SM groups, hypercharge included. This fixes the hypercharge of the bulk neutrino to be null. The straightforward output of this constraint is the restoration of the electric charge quantization. However, we should notice that on these theories $B-L$ does not seem to be an anomaly free symmetry, and thus, it can not be consistently gauged, at least within the minimal context we have considered along our discussion. This mark a clear difference with the four dimensional models. Further addition of other higher dimensional terms/fields to the action may be needed to insure anomaly cancellation. That would be the case of two extra dimensions, for instance, where antisymmetric tensor fields have to be added.

Acknowledgements. We would like to thank R.N. Mohapatra for comments. APL also wishes to thank R.N. Mohapatra and the University of Maryland particle theory group for the warm hospitality and support during the last stages of this work. The work of CP is supported by Fundação de Amparo à Pesquisa do Estado de São Paulo (FAPESP).

[1] O.Klein, Nature 118, 516 (1926).

[2] P.A.M. Dirac, Proc. R. Soc. A 133, 60 (1931).

[3] J.C. Pati and A. Salam, Phys. Rev. D 10, 275 (1974); H. Georgi and S.L. Glashow, Phys. Rev. Lett. 32, 438 (1974).

[4] R. Foot, G.C. Joshi, H. Lew and R.R Volkas, Mod. Phys. Lett A5, 2721 (1990); R. Foot, H. Lew and R.R Volkas, J. Phys. G 19, 269 (1993).

[5] K.S. Babu and R.N. Mohapatra, Phys. Rev. Lett. 63, 938 (1989); Phys. Rev. D 41, 271 (1990).

[6] K.S. Babu and R.N. Mohapatra, Phys. Rev. D 42, 3866 (1990).

[7] Y. Suzuki et al., Super-Kamiokande collaboration, Nucl. Phys. Proc. Suppl. 77, 35 (1999); Q. Ahmed et al, SNO collaboration, Phys. Rev. Lett. 87, 071301 (2001).

[8] Y. Fukuda et al., Phys. Rev. Lett. 81, 1562 (1998).

[9] C. Athanassopoulos et al., Phys. Rev. C54, 2685 (1996); A. Aguilar et al., hep-ex/0104049.

[10] N. Arkani-Hamed, S. Dimopoulos and G. Dvali, Phys. Lett. B429, 263 (1998); I. Antoniadis, S. Dimopoulos and G. Dvali, Phys. Lett. B436, 257 (1998).

[11] K. R. Dienes, E. Dudas and T. Gherghetta, Nucl. Phys. B557, 25 (1999); N. Arkani-Hamed, S. Dimopoulos, G. Dvali and J. March-Russell, hep-ph/9811448; G. Dvali and A.Y. Smirnov, Nucl. Phys. B563, 63 (1999).

[12] R. N. Mohapatra and A. Pérez-Lorenzana, Nucl. Phys. B593, 451 (2001).

[13] For further references see also: R. N. Mohapatra and A. Pérez-Lorenzana, Nucl. Phys. B576, 466 (2000); R. Barbieri, P. Creminelli and A. Strumia, Nucl.Phys. B585, 28 (2000); 
[14] H. Davoudiasl, P. Langacker and M. Perelstein, hep-ph/0201128.

[15] M. Gell-Mann, P. Ramond and R. Slansky, in Supergravity, eds. P. van Niewenhuizen and D.Z. Freedman (North Holland 1979); T. Yanagida, in Proceedings of Workshop on Unified Theory and Baryon number in the Universe, eds. O. Sawada and A. Sugamoto (KEK 1979); R. N. Mohapatra and G. Senjanović, Phys. Rev. Lett. 44, 912 (1980).

[16] C.D. Hoyle, et. al, Phys. Rev. Lett. 86, 1418 (2001).

[17] See for instance: G. F. Giudice, R. Rattazzi and J. D. Wells, Nucl. Phys. B544, 3 (1999); E. A. Mirabelli, M. Perelstein and Michael E. Peskin, Phys. Rev. Lett. 82, 2236 (1999); J. L. Hewett, Phys. Rev. Lett. 82, 4765 (1999); V. Barger, T. Han, C. Kao and R. J. Zhang, Phys. Lett. B461, 34 (1999); S. Hannestad, G.G. Raffelt, Phys. Rev. Lett. 88, 071301 (2002).

[18] N. Arkani-Hamed, A.G. Cohen, H. Georgi, Phys. Lett. B516, 395 (2001); C.A. Scrucca, M. Serone, L. Silvestrini, F. Zwirner, Phys. Lett. B525, 169 (2002). 